\title{
Maintaining Specific Natural Frequency of Damped System despite Mass Modification
}

\author{
Rui Zhu $\mathbb{D}^{1},{ }^{1}$ Qingguo Fei $\mathbb{D}^{1},{ }^{1}$ Dong Jiang, ${ }^{2,3}$ and Zhifu Cao ${ }^{3}$ \\ ${ }^{1}$ School of Mechanical Engineering, Southeast University, Nanjing 210096, China \\ ${ }^{2}$ School of Mechanical and Electronic Engineering, Nanjing Forestry University, Nanjing, 210037, China \\ ${ }^{3}$ Institute of Aerospace Machinery and Dynamics, Southeast University, Nanjing, 211189, China
}

Correspondence should be addressed to Qingguo Fei; qgfei@seu.edu.cn

Received 14 April 2019; Revised 5 September 2019; Accepted 17 September 2019; Published 3 November 2019

Academic Editor: Santiago Hernández

Copyright ( 2019 Rui Zhu et al. This is an open access article distributed under the Creative Commons Attribution License, which permits unrestricted use, distribution, and reproduction in any medium, provided the original work is properly cited.

In aerospace engineering, structural modifications play an essential role in design of structures. In some cases, it is necessary to guarantee that a specific natural frequency of the structure remains unchanged when additional masses are attached. The methods based on the Sherman-Morrison formula are proposed in this paper, called the optimal selection method and the absolute value method, to maintain the specific natural frequency. The methods are both implemented by installing a spring on the system and can eliminate the effect of the additional mass on the specific frequency. The proposed methods were verified to be effective and accurate through numerical simulations. Results show that the optimal selection method has similar applicability as the existing real value method, and both methods are applicable only in cases of small damping. In addition, the absolute value method has extensive applicability in systems with either small or large damping.

\section{Introduction}

The structural modification is an essential aspect in many engineering designs. Structural modification generally comprises mass, stiffness, and damping changes. During the design and operation phase $[1,2]$, structural modifications are as a common way to improve the dynamic characteristics of the original structure, such as the frequency control $[3,4]$. A common source of excessive vibration is at a natural frequency that coincides with or lies in the vicinity of an ambient vibration frequency $[5,6]$, which can cause structural failure [7]. When the original system has made a difference, preventing resonance or preserving a certain frequency of the system can avoid the requirement of designing the new controller.

Generally, the structural modification problem is classified into two categories: the direct structural modification problem [8] and the inverse structural modification problem $[9,10]$. The direct structural modification problem is aimed at predicting the effect of physical modifications on the dynamic behavior of the structure. The inverse structural modification problem involves determining the necessary adjustments so that the modified structure can possess the desired dynamic response.

In recent years, the inverse structural modification problem has drawn widespread attention. A method incorporating modal analysis for determining mass and stiffness modifications was developed for achieving the desired spectrum for target systems [11]. This method can overcome the difficulties resulting from the incomplete data and addresses an optimization problem instead of providing an exact solution. $\mathrm{Li}$ and $\mathrm{He}$ [12] developed an approach for structural modification of a dynamic system, wherein a set of linear equations were employed instead of an eigenvalue solution, thereby requiring the frequency response functions (FRFs) at merely the modification positions. Furthermore, Gürgöze and Inceo [13] determined the stiffness coefficient required for preserving the fundamental frequency of a beam structure under various supporting conditions to eliminate the effect of mass modification. A substructure-coupling concept [14] was utilized to obtain system equations. This can be applied to substantial model changes and get a considerable 
improvement in the natural frequencies. In other work, Mermertaş and Gürgöze [15] presented the impedance coupling method to preserve the fundamental frequency of the plates. The experiment results showed that the method is correct and valid. Joseph [16] presented an approach based on the solution of a partial inverse eigenvalue problem, and this method can be utilized to generate initial feasible designs for optimum design problems with frequency constraints. Olsson and Lidström [9] applied linear constraints on an undamped structure in an inverse manner to obtain designed modal properties. Çakar [17] developed the method based on the Sherman-Morrison (SM) formula [18] to determine the necessary spring stiffness, and further studies on weakly damped structures were conducted as well. There are some limitations in practical engineering application since this method had been only proved to be applicable for systems with low-level damping. Different from the existing techniques of freedom correction, a method [19] based on receptances was presented to achieve the modification by adding multiple mass-spring subsystems to the original structure. Two methods [20] were developed to prevent the shifting of the troublesome natural frequencies of an undamped system after mass modification. This study is interested in the determination of necessary stiffness modifications in order to keep a certain number of natural frequencies of the system unchanged despite mass attachments. Based on the concurrent use of the modification of the system mass and stiffness parameters, a control approach was proposed in the experimental application [8]. The allowable subspace was utilized to perform eigenstructure assignment in the vibration system. The advantage of the method is to overcome the limitations either passive modifications or active control alone. Herrada et al. [21] presented a method for accelerating parametric studies to account for local modifications in large finite element models.

Most of the existing research focuses on the structural modification of undamped structures, while limited study has been carried out on damped systems for structural modification. The current literature simply refers to the effect of damping, which is only suitable for the systems with lowlevel damping. Two methods are developed that installing the spring on the structure guarantees a certain natural frequency for the damped structure unchanged after the mass modification. The outline of the work is formulated as follows: The theoretical basis of two methods (the optimal selection method and the absolute value method) is introduced in Section 2. Numerical simulation is conducted by employing a six degrees of freedom (Six-DoFs) spring-mass system in Section 3.1 to verify the effectiveness of the approaches. The best spring location between the coordinates is determined in Section 3.2. The adaptability to systems with the different levels of damping is further investigated in Section 3.3. Subsequently, a six-bay truss is undertaken to verify the approaches in Section 3.4.

\section{Theory of Structural Modification}

A method has been applied to determine the necessary spring coefficient $k$ in the system in Ref. [17]. A specific natural fre- quency of the structure is kept unchanged after modifications. For a damped system, the spring coefficient required $k$ is a complex value in this method. In the process of maintaining a specific natural frequency, the spring coefficient takes the real part of $k$, ignoring the influence of the imaginary part. For the sake of description, this method is called "real method." It is only suitable for the systems with lowlevel damping. To improve the applicability of damped structures, we proposed two methods to determine the spring coefficient $k$ of the substituting calculation in this paper. These methods are the optimal selection method and the absolute value method, respectively.

2.1. Determine the Initial Spring Coefficient. A damped massspring system with $n$ degrees of freedom is illustrated in Figure 1, where mass $m^{*}$ represents the designed mass modification in the coordinate $t$. The system without additional mass $m^{*}$ is considered as the original system. $\mathbf{K}, \mathbf{M}$, and $\mathbf{C}$ are the stiffness, mass, and structural damping matrices of the original system, respectively. The dynamic stiffness matrix $\mathbf{Z}$ of the structure is written as

$$
\mathbf{Z}=\mathbf{K}-\omega^{2} \mathbf{M}+i \omega \mathbf{C},
$$

where $\omega$ is the circular frequency and $i$ is the imaginary unit. The matrix $\mathbf{Z}$ is defined with respect to those coordinates, say $q$.

For the original system, the natural frequencies are represented as

$$
g=f(\mathbf{M}, \mathbf{C}, \mathbf{K})
$$

which is the function of the matrices ( $\mathbf{K}, \mathbf{M}$, and $\mathbf{C})$.

The natural frequencies of the structure tend to decrease when the extra mass $m^{*}$ is added to the system. The updated frequencies of the modified system are represented as

$$
g^{*}=f\left(\mathbf{M}+m^{*}, \mathbf{C}, \mathbf{K}\right) \text {. }
$$

To guarantee a certain natural frequency unchanged after mass modification, the spring is added on the original structure. There are two ways. One is that a spring $k_{r e}^{*}$ is added between some predefined coordinates $r$ and $e$. The other is that a grounded spring $k_{r r}^{*}$ is added in the coordinate $r$ in Figure 2. They both remove the frequency shifting effects caused by the mass $m^{*}$. At this time, the natural frequencies of the structure after spring modification can be represented as

$$
g^{* *}=f\left(\mathbf{M}+m^{*}, \mathbf{C}, \mathbf{K}+k_{r e}^{*}\right)
$$

The key point is to determine the coefficient of the additional spring. For practical engineering application, it is difficult to obtain the matrices $\mathbf{M}, \mathbf{C}$, and $\mathbf{K}$ of the structure accurately. However, the FRFs of the structure can be obtained through experimental measurement directly. When 


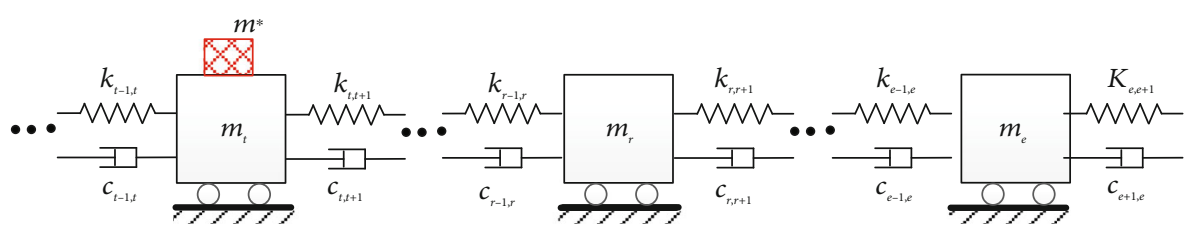

Figure 1: Damped spring-mass system with $n$ DoFs modified by mass.

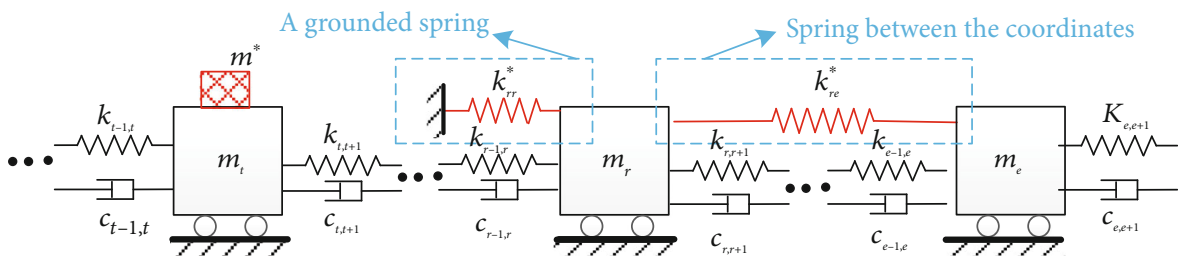

FIGURE 2: Damped spring-mass system with $n$ DoFs modified by mass and a spring.

the structure has modification, the change of the dynamic stiffness matrix can be represented as

$$
\Delta \mathbf{Z}=\frac{\partial \mathbf{Z}}{\partial q} d q
$$

where $\partial$ should be considered as the partial derivative of $Z$ with respect to all the coordinates.

The dynamic stiffness matrix $\mathbf{Z}^{*}$ of the modified system can be described as

$$
\mathbf{Z}^{*}=\mathbf{Z}+\frac{\partial \mathbf{Z}}{\partial q} d q
$$

According to the relationship between the receptance matrix and the dynamic stiffness matrix, the receptance can be obtained by

$$
\begin{aligned}
\boldsymbol{\alpha} & =\mathbf{Z}^{-1}, \\
\boldsymbol{\alpha}^{*} & =\mathbf{Z}^{*-1} .
\end{aligned}
$$

If $\Delta \mathbf{Z}=\{u\}\{v\}^{T}$, where $\{u\}$ and $\{v\}$ are column vectors, the FRFs of the modified structure can be calculated by using the SM formula:

$$
\boldsymbol{\alpha}^{*}=\mathbf{Z}^{*-1}=\boldsymbol{\alpha}-\frac{\boldsymbol{\alpha}\{u\}\{v\}^{T} \boldsymbol{\alpha}}{1+\{v\}^{T} \boldsymbol{\alpha}\{u\}} .
$$

The necessary stiffness coefficient can be determined conclusively based on the SM formula. The specific derivation process is as follows.

For the initial system, $\alpha_{r e}$ is the receptance; the subscripts $r$ and $e$ are the response and excitation coordinates, respectively. When the mass $m^{*}$ is added for the design aim, the receptance is represented by $\alpha_{r e}^{*}$. To remove the shifting effect of the mass $m^{*}, k_{r e}^{*}$ can be added between coordinates $r$ and $e$. Here, the dynamic stiffness matrix is represented by $\mathbf{Z}^{* *}$, and the receptance is represented by $\alpha_{r e}^{* *}$. The change between $\mathbf{Z}^{*}$ and $\mathbf{Z}^{* *}$ can be represented by $\Delta \mathbf{Z}^{*}$ caused by the stiffness coefficient $k_{r e}^{*}$. For the stiffness modification, the corresponding modification vectors $\left\{u^{*}\right\}$ and $\left\{v^{*}\right\}$ are written by

$$
\begin{aligned}
& \left\{u^{*}\right\}=\left\{\begin{array}{lllll}
\cdots & -1 & \cdots & 1 & \cdots
\end{array}\right\}^{T}, \\
& \left\{v^{*}\right\}=\left\{\begin{array}{llllll}
\cdots & -k_{r e}^{*} & \cdots & k_{r e}^{*} & \cdots
\end{array}\right\}^{T},
\end{aligned}
$$

where other elements of the vectors $\left\{u^{*}\right\}$ and $\left\{v^{*}\right\}$ are all zero except the coordinate $r$ and the coordinate $e$. Substituting Equation (9) and Equation (10) into Equation (8), the relationship between $\alpha_{r e}^{*}$ and $\alpha_{r e}^{* *}$ can be obtained by

$$
\alpha_{r e}^{* *}=\frac{\alpha_{r e}^{*}+k_{r e}^{*}\left(\alpha_{r r}^{*} \alpha_{e e}^{*}-\alpha_{r e}^{* 2}\right)}{1+k_{r e}^{*}\left(\alpha_{r r}^{*}-2 \alpha_{r e}^{*}+\alpha_{e e}^{*}\right)},
$$

where the superscript $(*)$ of $\alpha_{r e}^{*}$ shows the mass modification, and $\alpha_{r e}^{* *}$ contains the effects of the mass modification and the stiffness modification. In the modal test, the acceleration signal is generally more sensitive and can be better measured. According to the relationship of acceleration admittance and displacement admittance $\left(\mathbf{h}=-\omega^{2} \boldsymbol{\alpha}\right)$, it is realizable to acquire the acceleration admittance $h_{r e}^{* *}$ using Equation (11):

$$
h_{r e}^{* *}=\frac{-\omega^{2} h_{r e}^{*}+k_{r e}^{*}\left(h_{r r}^{*} h_{e e}^{*}-h_{r e}^{* 2}\right)}{-\omega^{2}+k_{r e}^{*}\left(h_{r r}^{*}-2 h_{r e}^{*}+h_{e e}^{*}\right)} .
$$

When a grounded spring $k_{r r}^{*}$ is added in coordinate $r$ to compensate the shifting effect of the added mass on a specific natural frequency, the corresponding modification vectors $\left\{u^{*}\right\}$ and $\left\{v^{*}\right\}$ can be written by

$$
\begin{aligned}
& \left\{u^{*}\right\}=\left\{\begin{array}{lll}
\cdots & 1 & \cdots
\end{array}\right\}^{T}, \\
& \left\{v^{*}\right\}=\left\{\begin{array}{lll}
\cdots & k_{r r}^{*} & \cdots
\end{array}\right\}^{T},
\end{aligned}
$$


where other elements of the vectors $\left\{u^{*}\right\}$ and $\left\{v^{*}\right\}$ are all zero except coordinate $r$. The corresponding Equation (12) can be written as

$$
h_{r r}^{* *}=\frac{-\omega^{2} h_{r r}^{*}}{-\omega^{2}+k_{r r}^{*} h_{r r}^{*}} .
$$

Next, how to determine the spring $k_{r e}^{*}$ is introduced. To guarantee the specific natural frequency $\left(\omega_{o}\right)$ of the structure unchanged after the mass and spring modifications, the amplitude curve of the FRF $h_{r e}^{* *}$ should have a maximum local value at the frequency $\omega_{0}$. In other words, the denominator of Equation (12) is equal to zero:

$$
-\omega^{2}+k_{r e}^{*}\left(h_{r r}^{*}-2 h_{r e}^{*}+h_{e e}^{*}\right)=0, \quad \omega=\omega_{o} .
$$

Then, the necessary spring coefficient $k_{r e}^{*}$ can be calculated by Equation (15):

$$
k_{r e}^{*}=\frac{\omega_{o}^{2}}{h_{r r}^{*}-2 h_{r \mathrm{e}}^{*}+h_{e e}^{*}} .
$$

When the system is undamped, the necessary spring coefficient $k_{r e}^{*}$ is the real value in Equation (16). However, the actual structure is damped, and the FRF is a complex function. Then, the denominator of Equation (16) is a complex value, and corresponding $k_{r e}^{*}$ is a complex stiffness. The actual spring stiffness should be positive. It is impossible to use $k_{r e}^{*}$ in Equation (16) directly. To determine the effective stiffness, the optimal selection method and the absolute value method are proposed.

2.2. Optimal Selection Method. A method called the optimal selection is proposed. The main idea of the method is that the problem of determining the effective stiffness problem is equivalent to the problem of solving the minimum value of the quadratic equation with one unknown.

As known in the engineering application, the spring stiffness $k_{r e}^{*}$ needed to compensate the effect of the mass $m^{*}$ at $\omega_{o}$ should be positive. In Equation (12), both the numerator and the denominator change on the right side of the equation when $k_{r e}^{*}$ changes. Assume that the influence of stiffness on the numerator is ignored, only the impact on the denominator is considered. To keep the certain natural frequency $\left(\omega_{o}\right)$ unchanged, the amplitude curve of the FRF $h_{r e}^{* *}$ should have a maximum local value at $\omega_{0}$. With ignoring the stiffness influence on the numerator, the square of the module of the denominator in Equation (12) should reach a minimum at $\omega_{0}$.

At the frequency $\omega_{0}$, the parenthesis item in the denominator of Equation (12) is known as a complex, which can be represented by $(a+b i)$, and $a$ and $b$ are real numbers. When the natural frequency is $\omega_{o}$, the denominator can be written by

$$
W\left(k_{r e}^{*}\right)=\left(k_{r e}^{*} a-\omega_{o}^{2}\right)+k_{r e}^{*} b i .
$$

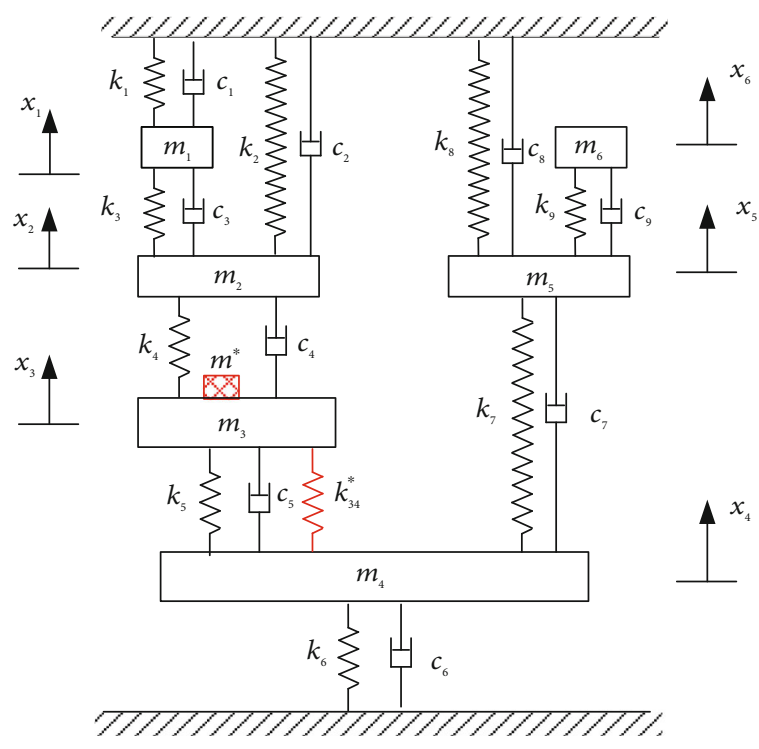

FIgURE 3: Six-DoFs damped mass-spring system with added mass and spring.

TABle 1: Natural frequencies of the original system and the modified system.

\begin{tabular}{lcccccc}
\hline Mode order $(r)$ & 1 & 2 & 3 & 4 & 5 & 6 \\
\hline$\omega_{r}$ & 0.109 & 0.145 & 0.205 & 0.261 & 0.314 & 0.337 \\
$\omega^{*}{ }_{r}$ & 0.106 & 0.133 & 0.199 & 0.254 & 0.310 & 0.330 \\
Error $(\%)$ & 2.75 & 8.28 & 2.93 & 2.68 & 1.27 & 2.08 \\
\hline
\end{tabular}

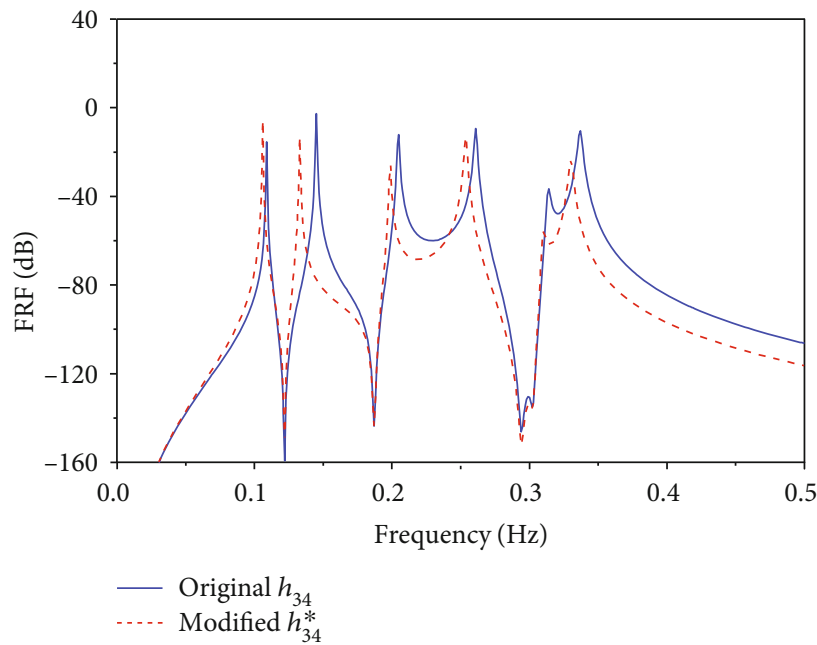

FIgURE 4: Comparison of exact and modified FRF $h_{34}$ after mass modification.

The square of the module of $W$ can be expressed as

$$
F\left(k_{r e}^{*}\right)=\left(a^{2}+b^{2}\right) k_{r e}^{* 2}-2 a \omega_{o}^{2} k_{r e}^{*}+\omega_{o}^{4},
$$

where function $F$ is a quadratic equation with one unknown and $k_{r e}^{*}$ is the real independent variable. From the above analysis, the special frequency $\left(\omega_{0}\right)$ of the structure can be 
TABLE 2: Necessary spring coefficient $(\mathrm{N} / \mathrm{m})$ and the natural frequencies.

\begin{tabular}{|c|c|c|c|c|c|c|c|}
\hline \multirow{2}{*}{ Methods } & \multirow{2}{*}{ Spring coefficient } & \multicolumn{6}{|c|}{ Natural frequencies $(\mathrm{Hz})$} \\
\hline & & 1 & 2 & 3 & 4 & 5 & 6 \\
\hline Original & & 0.109 & 0.145 & 0.205 & 0.261 & 0.314 & 0.337 \\
\hline Real method & 2.327 & 0.106 & 0.145 & 0.198 & 0.283 & 0.310 & 0.430 \\
\hline Optimal selection method & $k_{34(\mathrm{opt})}^{*}=2.327$ & 0.106 & 0.145 & 0.198 & 0.283 & 0.310 & 0.430 \\
\hline Absolute value method & $k_{34(\mathrm{abs})}^{*}=2.333$ & $0.106(2.8 \%)$ & $0.145(0.0 \%)$ & $0.199(3.4 \%)$ & $0.288(8.4 \%)$ & $0.310(1.3 \%)$ & $0.430(27.6 \%)$ \\
\hline
\end{tabular}

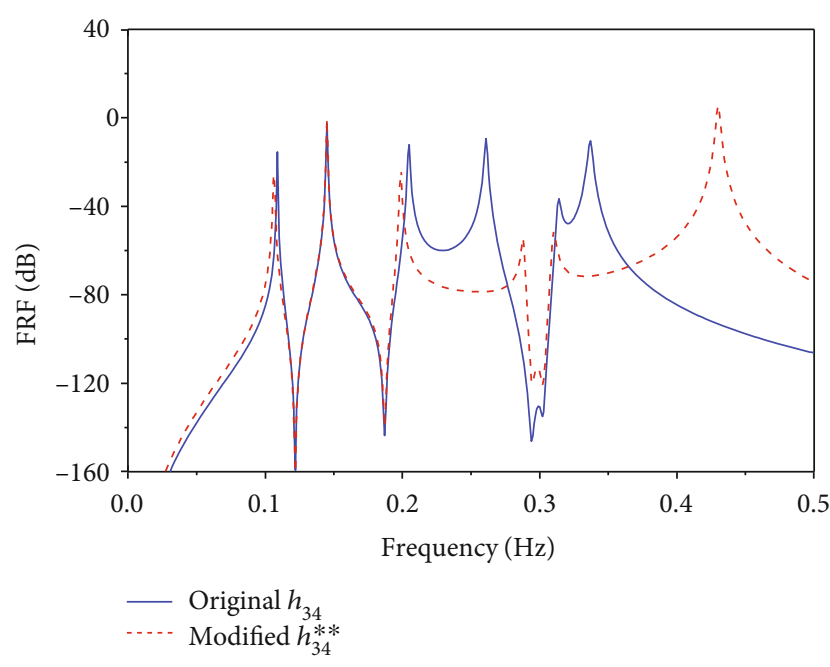

FIGURE 5: Comparison of exact and modified FRF $h_{34}$ after the mass and stiffness modification.

TABLE 3: Necessary spring coefficient $k_{i j(\mathrm{opt})}^{*}(\mathrm{~N} / \mathrm{m})$.

\begin{tabular}{lcccccc}
\hline $\begin{array}{l}\text { Coordinate } j \\
\text { Coordinate } i\end{array}$ & 1 & 2 & 3 & 4 & 5 & 6 \\
\hline 1 & -2.533 & -1.189 & -0.897 & -0.595 & 4.326 & 0.197 \\
2 & - & 1.582 & -1.608 & -1.144 & 0.766 & 0.155 \\
3 & - & - & 0.415 & 2.327 & 0.312 & $\mathbf{0 . 1 1 2}$ \\
4 & - & - & - & -47.982 & 3.801 & 0.294 \\
5 & - & - & - & - & 3.712 & 1.882 \\
6 & - & - & - & - & - & 0.292 \\
\hline
\end{tabular}

guaranteed when function $F$ reaches the minimum value. The minimum amount of $F$ is on the symmetry axis, so the necessary stiffness coefficient can be determined by

$$
k_{r e(\mathrm{Opt})}^{*}=\frac{a \omega_{o}^{2}}{a^{2}+b^{2}} .
$$

From the perspective of optimal selection, the stiffness of the upper calculation in Equation (19) is added to the damped system, which can ensure the specific frequency unchanged.

2.3. Absolute Value Method. Different from neglecting the effect of the imaginary part of stiffness in the existing method above, a method of considering the imaginary part is proposed, namely, the absolute value method. It is known that when the structure is a damped system, $k_{r e}^{*}$ is a complex number in Equation (16). For complex numbers, two main characteristics are the modulus and phase of the complex number.

In numerical calculation, the imaginary part of $k_{r e}^{*}$ cannot be omitted. From the physical point of view, the spring stiffness is the real number without the imaginary part. Considering views above at the same time, the concept of complex modulus is adopted. The desired spring coefficient can be obtained directly from the absolute value of $k_{r e}^{*}$ in Equation (16):

$$
k_{\mathrm{abs}}^{*}=\left|k_{r e}^{*}\right|=\frac{\omega_{o}^{2}}{\left|h_{r r}^{*}\left(\omega_{o}\right)-2 h_{r e}^{*}\left(\omega_{o}\right)+h_{e e}^{*}\left(\omega_{o}\right)\right|} .
$$

In this method, the desired spring coefficient not only considers the imaginary part of the initial stiffness but also guarantees the real number to meet the actual engineering requirements.

\section{Numerical Case Studies}

To verify the effectiveness of the proposed methods, numerical simulations are conducted by the Six-DoFs spring-mass system and six-bay truss. The best spring location of the Six-DoFs damped system between the coordinates is discussed. For further study on the applicability of methods, the structure with high-level damping is investigated.

3.1. Case 1: Six-DoFs Spring-Mass System. For the simulations, a six degrees of freedom model consisting of six concentrated masses attached to nine translational springs is considered as shown in Figure 3. The system parameters are $m_{i}=1 \mathrm{~kg}(i=1,2, \cdots, 6), k_{i}=1 \mathrm{~N} / \mathrm{m}(i=1,2, \cdots, 9)$, and $c_{i}=0.005 \mathrm{Ns} / \mathrm{m} \quad(i=1,2, \cdots, 9)$. For the design aim, a lumped mass $m^{*}$ of $0.5 \mathrm{~kg}$ is added as the mass modification in coordinate 3 .

There are discernible changes in the natural frequencies of the structure due to the attachment of the additional mass. The natural frequencies of the modified system and the original system are shown in Table 1. $\omega_{r}$ represents the natural frequency of the original system, and $\omega^{*}{ }_{r}$ represents the natural frequency of the modified system. As is known, the natural frequencies of the system decrease due to mass modification. The errors of the natural frequencies of the modified system relative to the original system are shown in the fourth line of Table 1. Meanwhile, the transfer acceleration admittance $h_{34}$ of the original and modified systems is compared in Figure 4. As expected, the resonance frequencies 
TABLE 4: Stiffness values of different methods under different modal damping ratios.

\begin{tabular}{|c|c|c|c|c|c|}
\hline \multirow{2}{*}{$\begin{array}{l}\text { Cases } \\
\text { Methods }\end{array}$} & \multicolumn{5}{|c|}{ Modal damping ratio of the second natural frequency (\%) } \\
\hline & 0.84 & 1.66 & 2.52 & 3.39 & 4.24 \\
\hline Initial stiffness & $2.202-0.329 i$ & $1.871-1.129 i$ & $1.464-1.432 i$ & $1.075-1.574 i$ & $0.745-1.614 i$ \\
\hline Real method & 2.202 & 1.871 & 1.464 & 1.075 & 0.745 \\
\hline Optimal selection method & 2.202 & 1.871 & 1.464 & 1.075 & 0.745 \\
\hline Absolute value method & 2.292 & 2.185 & 2.048 & 1.906 & 1.777 \\
\hline
\end{tabular}

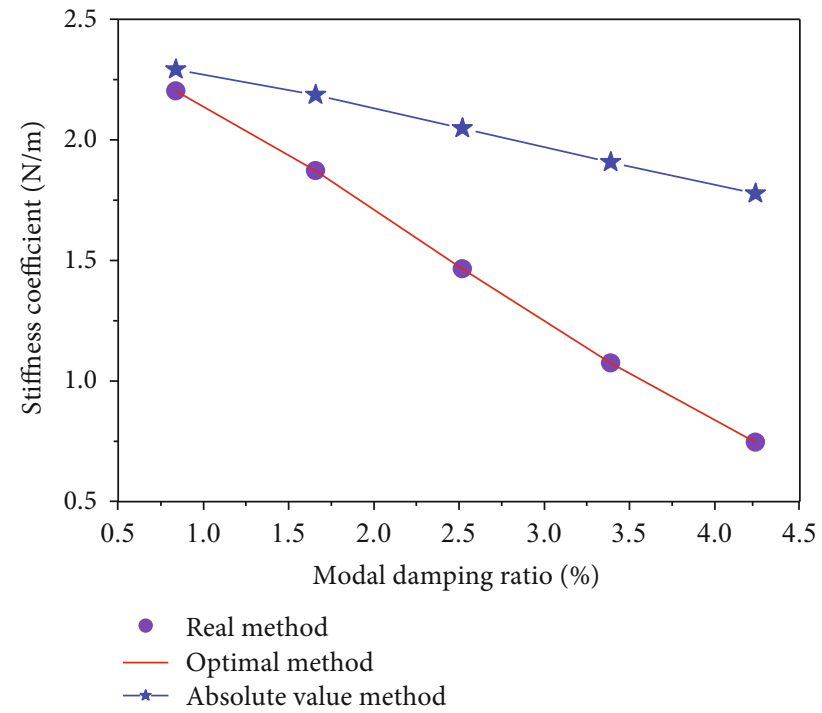

FIgURE 6: Stiffness values under different modal damping ratios.

TABLE 5: Second frequency and error under different modal damping ratios.

\begin{tabular}{lccccc}
\hline Cases & \multicolumn{5}{c}{ Modal damping ratio of the second } \\
Methods & 0.84 & 1.66 & 2.52 & 3.39 & 4.24 \\
\hline Real method & & & & \\
Frequency (Hz) & 0.1447 & 0.1440 & 0.1438 & 0.1435 & 0.1397 \\
Error (\%) & 0.178 & 0.69 & 0.82 & 1.05 & 3.66 \\
Optimal selection method & & & & & \\
Frequency (Hz) & 0.1447 & 0.1440 & 0.1438 & 0.1435 & 0.1397 \\
Error (\%) & 0.178 & 0.69 & 0.82 & 1.05 & 3.66 \\
Absolute value method & & & & & \\
Frequency (Hz) & 0.1449 & 0.1447 & 0.1444 & 0.1441 & 0.1438 \\
Error (\%) & 0.06 & 0.20 & 0.40 & 0.62 & 0.83 \\
\hline
\end{tabular}

of the system after mass modification are lower than those of the original system. The maximum error occurs at the 2nd order frequency (8.28\%). And the frequency errors of other orders are $2.75 \%$ (1st order), $2.93 \%$ (3rd order), $2.68 \%$ (4th order), $1.27 \%$ (5th order), and $2.08 \%$ (6th order), respectively.

Due to the obvious change of the second order after mass modification, this frequency is desired to be kept constant. To satisfy the desire above, decreasing the natural frequency should be compensated by stiffness modification. In practical

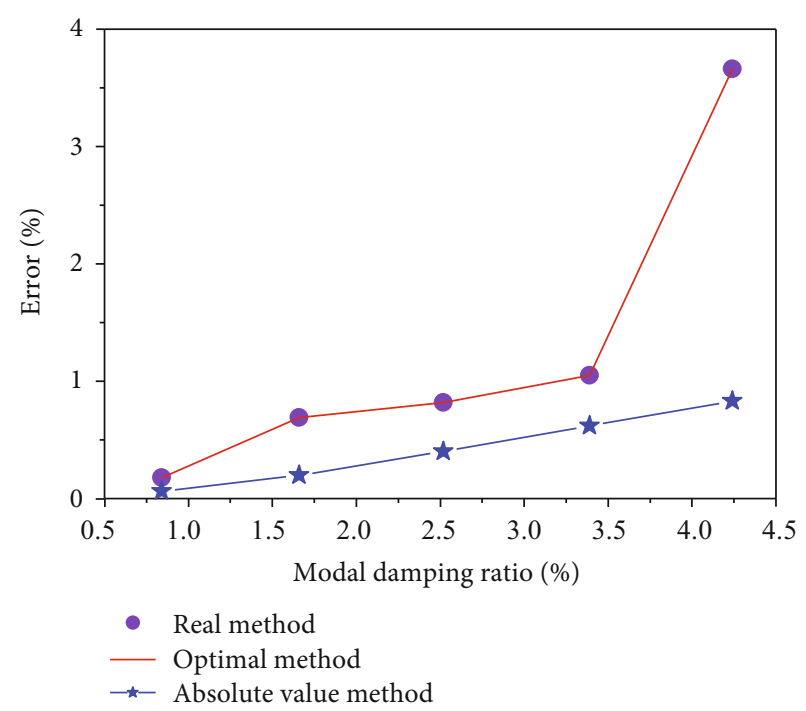

FIGURE 7: The errors of the three methods.

engineering application, there is a limited number of the spring installation position because of the design limitations. For illustration, let us assume, the position between the coordinate 3 and the coordinate 4 is regarded as the predefined location for the attachment of the spring. The stiffness coefficient of the spring is represented by $k_{34}^{*}$. The optimal selection method, the absolute value method, and the real method are used to determine the spring stiffness, respectively.

Based on the SM formula, the value of the initial spring coefficient is $k_{34}^{*}=2.327-0.162 i$ by using Equation (16). The stiffness determined by the three methods above is given in Table 2, respectively. Results show that the spring coefficient $k_{34(\mathrm{opt})}^{*}=2.327$ is very close to $k_{34(\mathrm{abs})}^{*}=2.333$. And the spring coefficient determined by the real method is equal to the one determined by the optimal selection method. Meanwhile, the results of the three methods after the stiffness modification are consistent.

As shown in Table 2, the desired and achieved natural frequencies were shown in bold. The second natural frequency of the system after the stiffness modification keeps unchanged $(0.145 \mathrm{~Hz})$, and other natural frequencies change.

After the stiffness modification, the acceleration FRF of the modified structure is calculated. The exact and modified FRF $h_{34}$ are shown in Figure 5. Results show that the FRF of the modified system near the second-order frequency range agrees well with the FRF of the original system. And the second natural frequency of the modified system is unchanged despite mass attachment by using the three 


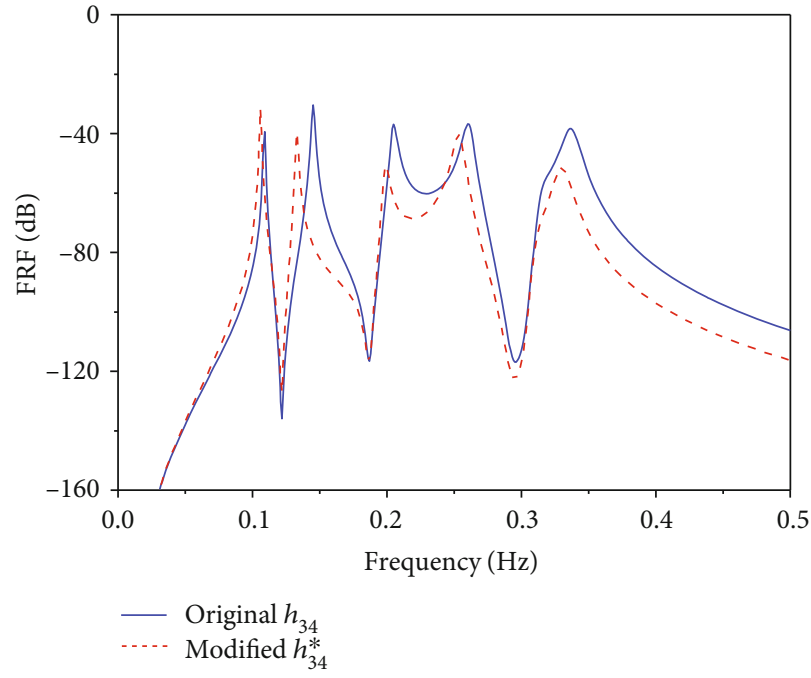

(a) Exact and modified FRFs after the mass modification

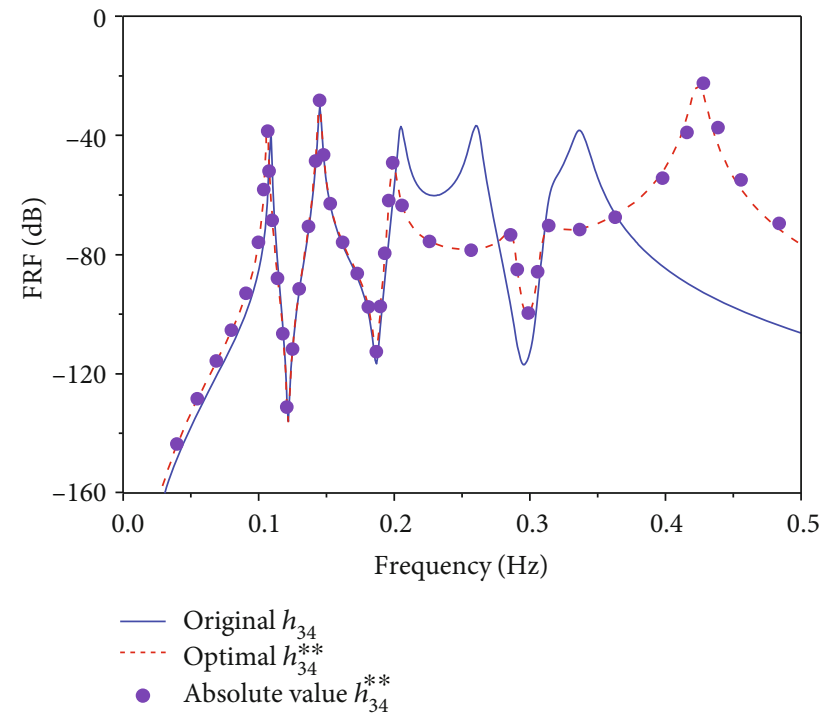

(b) Comparison of three methods

FIGURE 8: Comparison of FRFs between original and modified systems with modal damping ratio $0.84 \%$.

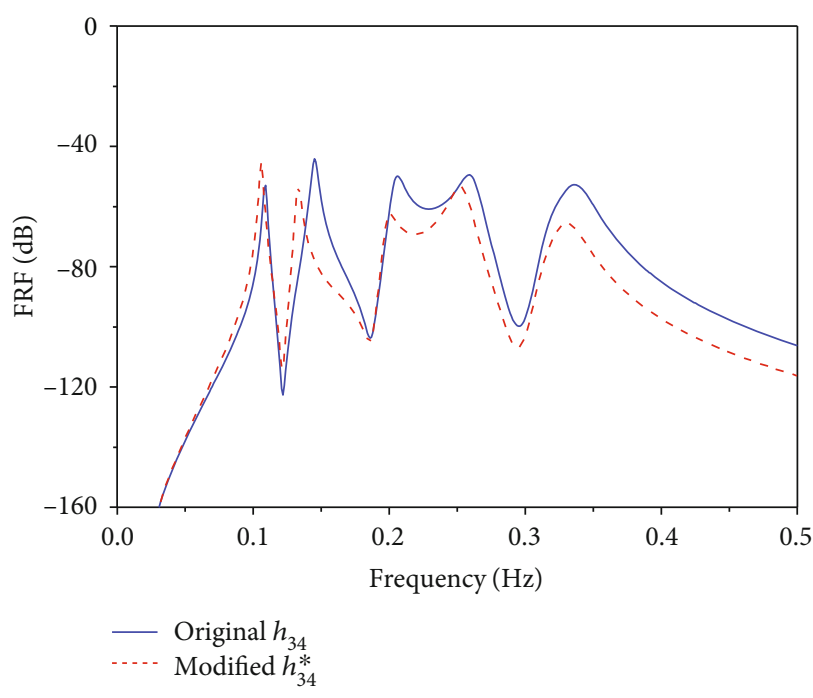

(a) Exact and modified FRFs after the mass modification

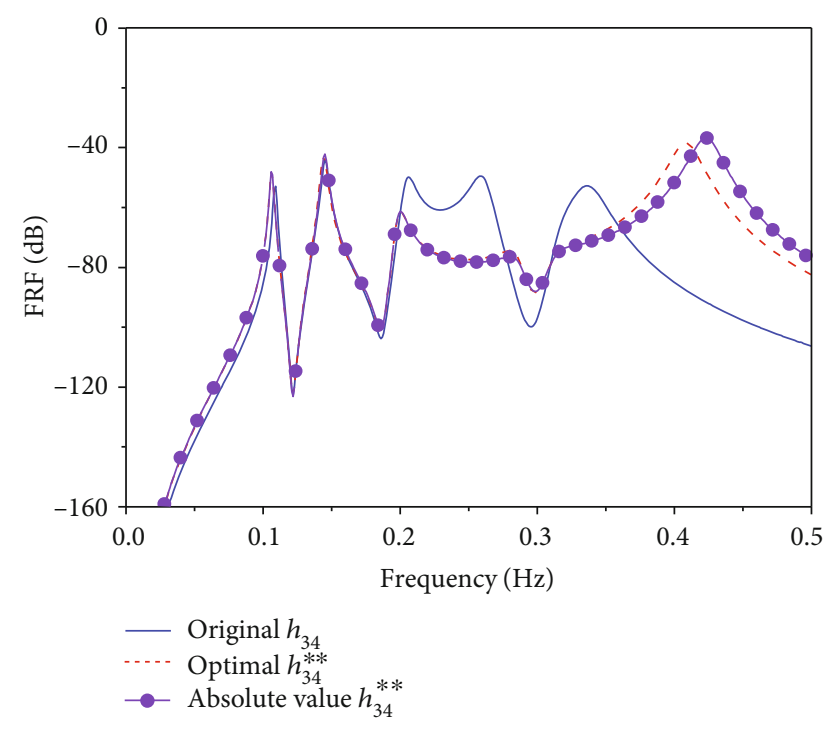

(b) Comparison of three methods

FIGURE 9: Comparison of FRFs between original and modified systems with modal damping ratio 1.66\%.

different methods above. Consequently, the efficiency of the two proposed methods is validated.

3.2. Case 2: The Best Spring Location. In this section, the aim is to determine the best spring location between all the coordinates. Necessary spring coefficients obtained by the optimal selection method are presented in Table 3. As known, the added spring $k_{i j}^{*}$ is equal to $k_{j i}^{*}$. Results show that it is not valid that some $k_{i j(\mathrm{opt})}^{*}$, such as $k_{12(\mathrm{opt})}^{*}=-1.189$, is negative because theoretically the spring stiffness should be positive. It is assumed that the optimal position is the one with the minimum spring stiffness. It can be concluded that the best position for spring installation is between the coordinates 3 and $6\left(k_{36(\mathrm{opt})}^{*}=0.112\right)$.

3.3. Case 3: Damping Study. In Ref. [17], the real method is only acceptable for the system with small damping and not acceptable for the system with high-level damping. The applicability of the proposed methods in this paper to the structure with high-level damping is investigated. The research object is still the same spring-mass system shown in Figure 3. Different damping coefficients are considered from 0.02 to $0.10 \mathrm{~N} / \mathrm{m}$, where the interval is 0.02 and there are five cases. 


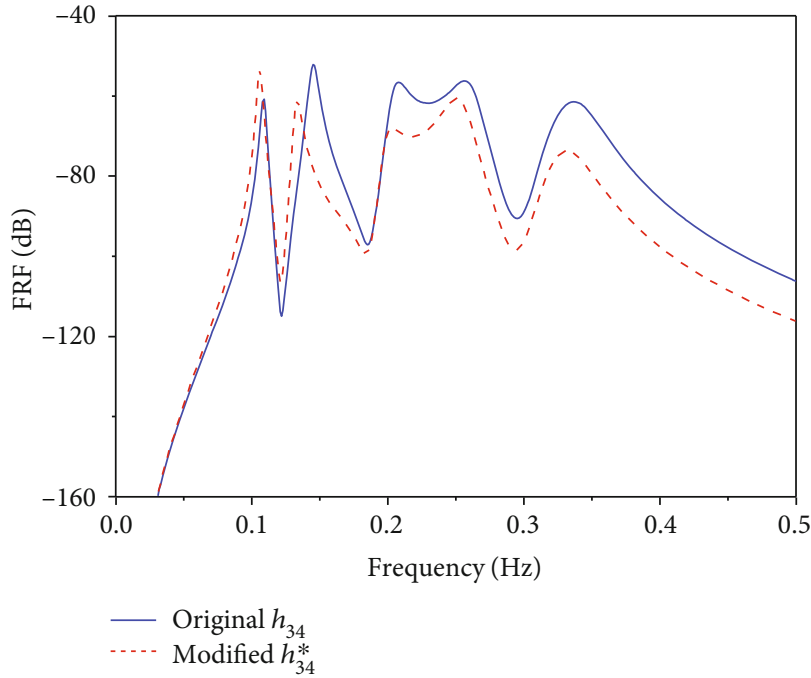

(a) Exact and modified FRFs after the mass modification

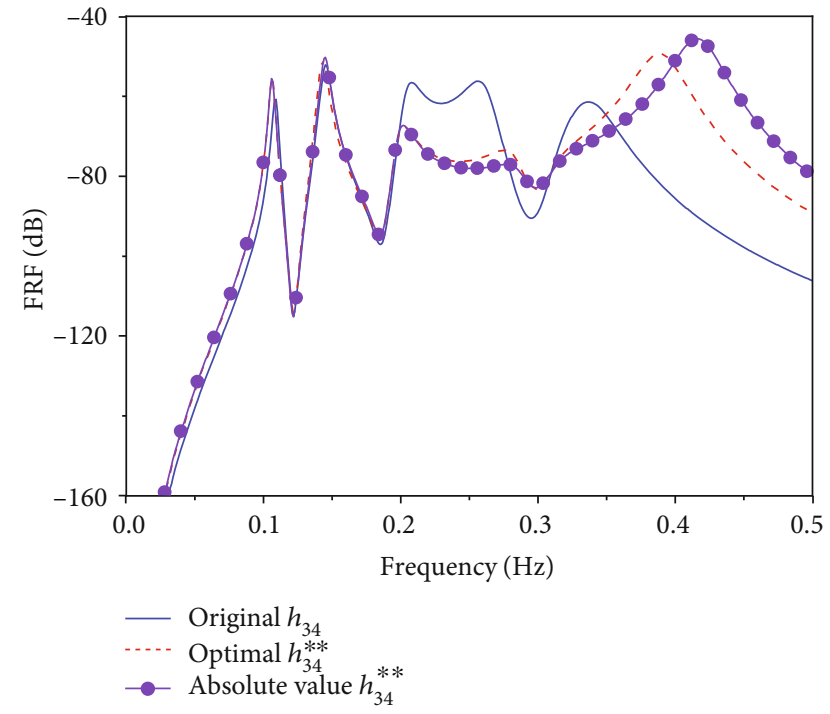

(b) Comparison of three methods

FIGURE 10: Comparison of FRFs between original and modified systems with modal damping ratio $2.52 \%$.

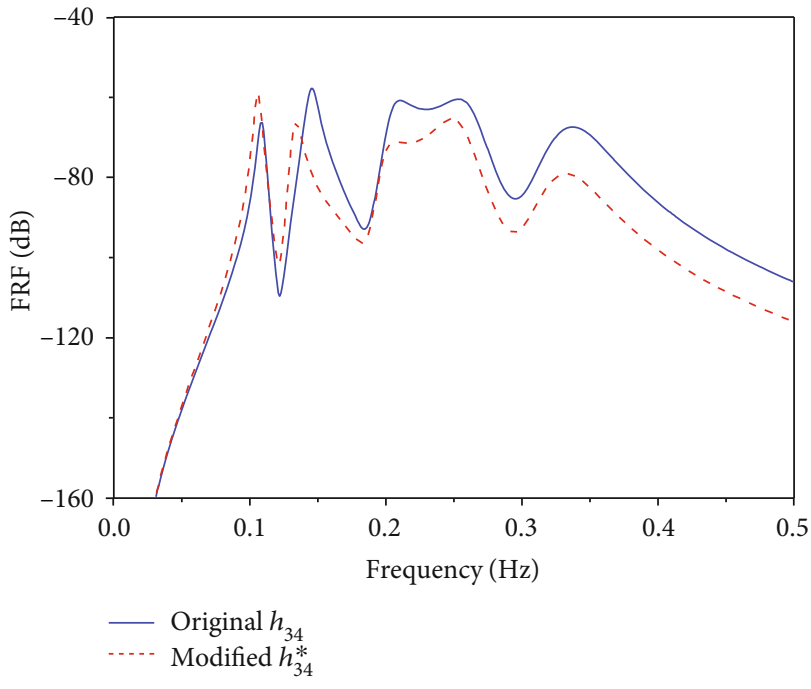

(a) Exact and modified FRFs after the mass modification

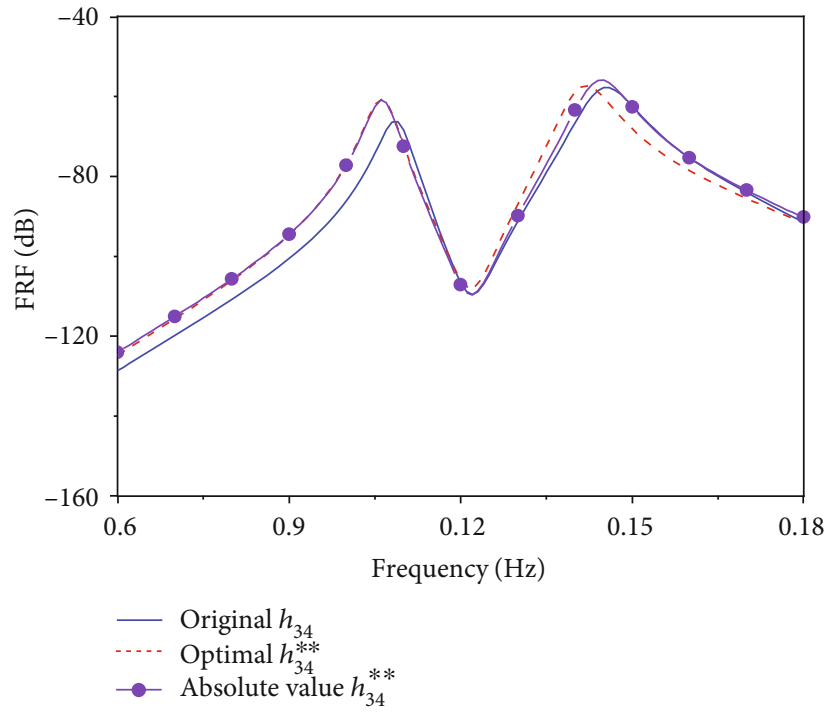

(b) Comparison of three methods

FIGURE 11: Comparison of FRFs between original and modified systems with modal damping ratio 3.39\%.

As is seen in Table 4, the initial stiffness by using Equation (16) is shown in the fifth line. For the different damping cases, the modal damping ratio of the corresponding secondorder frequency is given in the second line of Table 4. Results show that with the increase of the modal damping ratio, (i) the real part and the imaginary part of the initial stiffness both decrease; (ii) the stiffness values of all methods all reduce, and the spring coefficient determined by the real method is equal to the one determined by the optimal selection method; and (iii) the reduction of the stiffness value determined by the absolute value method is smaller than that of the optimal selection method shown in Figure 6.

After mass and stiffness modification, the error relative to $0.145 \mathrm{~Hz}$ and the second frequency are given in Table 5. The errors of three methods are given in Figure 7 so that the precision of the three methods are compared. Meanwhile, the acceleration FRFs of the modified structure after the stiffness modification are calculated and compared with those of the original for different cases in Figures 8-12.

As can be seen in Figure 7, the error of the real method (optimal selection method) is larger than the absolute method under the same damping condition. In the case of a low modal damping ratio (1\%), the error of the real method is small. However, the error increases significantly with the increase of the modal damping ratio; especially when the modal damping ratio reaches $4.24 \%$, the error has reached $3.6 \%$. Results show that the real method is only acceptable for the system with small damping and not acceptable for 


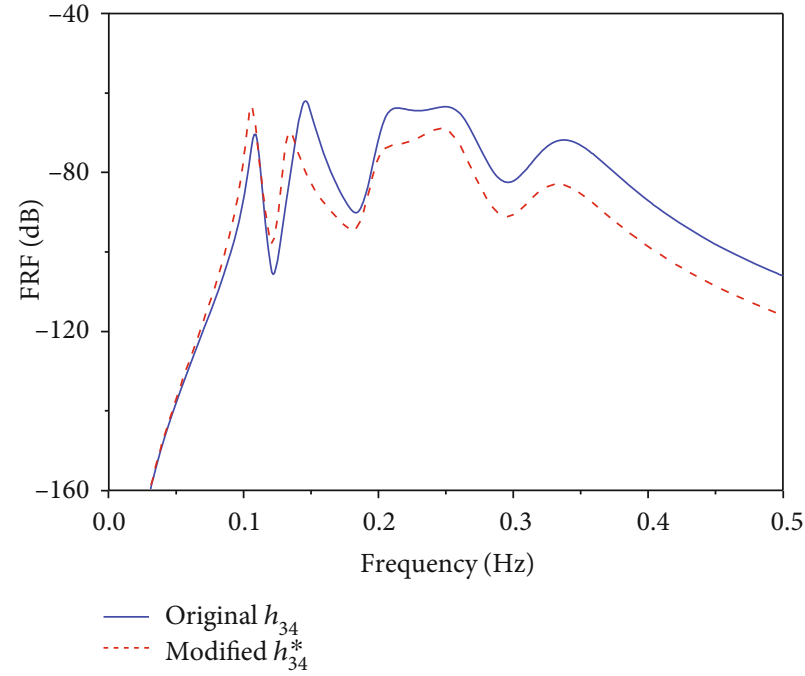

(a) Exact and modified FRFs after the mass modification

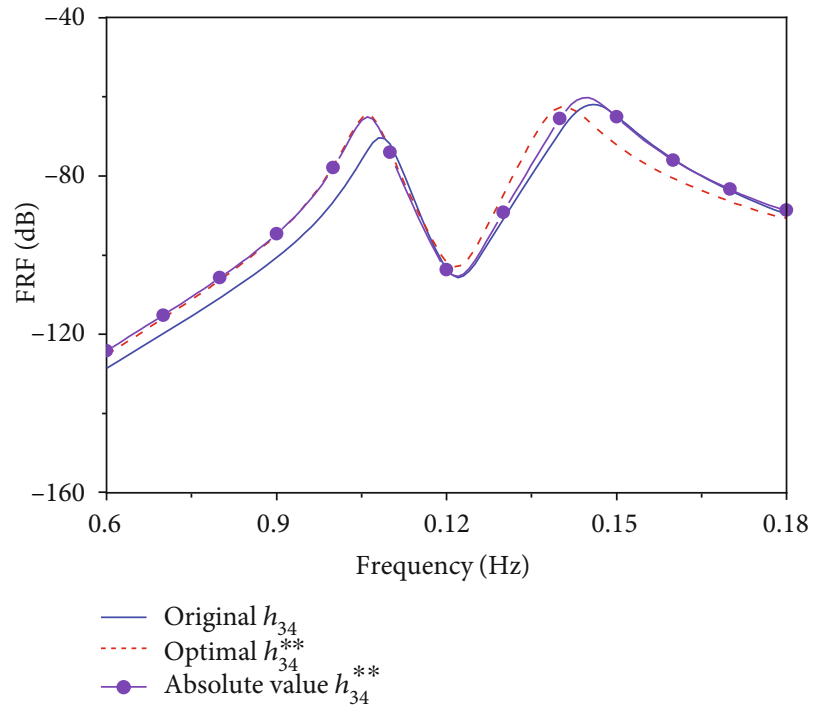

(b) Comparison of three methods

FIGURE 12: Comparison of FRFs between original and modified systems with modal damping ratio 4.24\%.

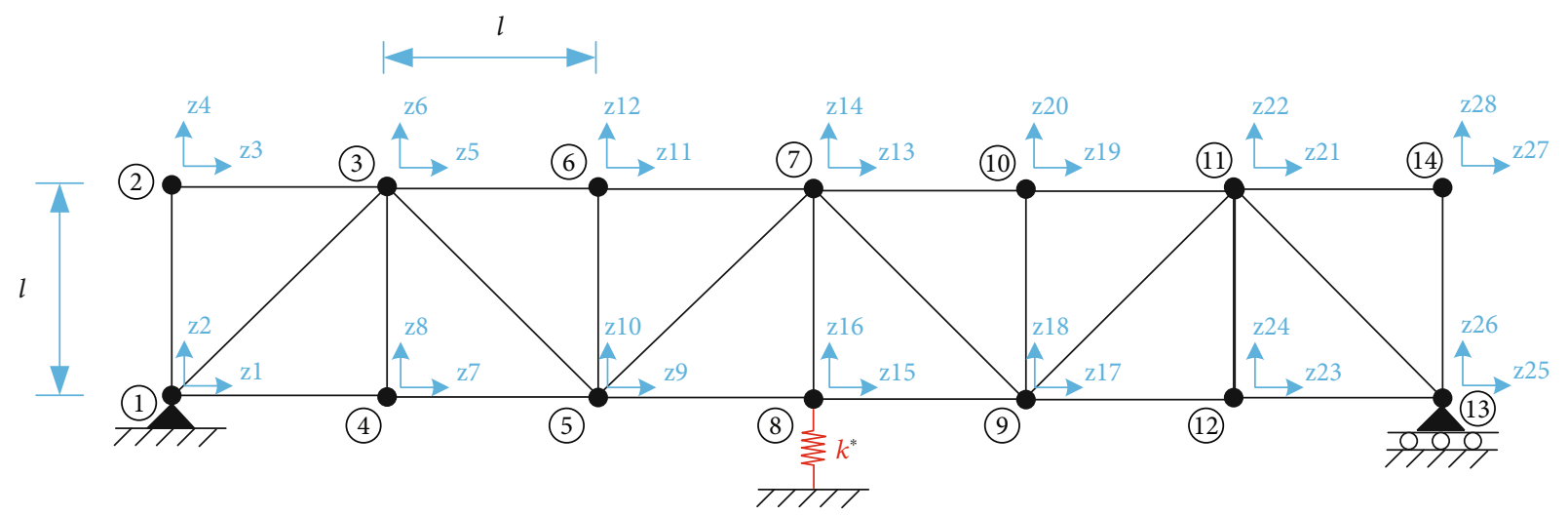

FIGURE 13: Planar truss system.

TABle 6: Natural frequencies of the original system and the modified system.

\begin{tabular}{lccccc}
\hline Mode order $(r)$ & 1 & 2 & 3 & 4 & 5 \\
\hline$\omega_{r}$ & 38.68 & 78.87 & 117.16 & 175.71 & 195.51 \\
$\omega^{*}{ }_{r}$ & 36.06 & 76.03 & 106.53 & 159.26 & 177.10 \\
Error $(\%)$ & 6.75 & 3.60 & 9.07 & 9.37 & 9.42 \\
\hline
\end{tabular}

the system with high-level damping. Meanwhile, the error of the absolute value method is still relatively small though the high-level damping. It is obvious that the absolute value method has outstanding performance. The difference between the results of the two methods is that the real method ignores the effect of the imaginary part, while the absolute method considers the factor of the imaginary part. When the damping is larger, the effect of the imaginary part is particularly important.

3.4. Case 4: Six-Bay Truss. A six-bay truss is investigated in Figure 13, which has 25 truss elements and 28 degrees of free- dom (DoFs). The cross-sectional area of each truss element is $A_{i}=1 \times 10^{-4} \mathrm{~m}^{2}$, Young's modulus for the truss-element material is $E=7 \times 10^{10} \mathrm{~Pa}$, the density of the truss-element material is $\rho=2700 \mathrm{~kg} / \mathrm{m}^{3}$, and the geometric parameter is $l=1.5 \mathrm{~m}$. The global DoFs of the joints are denoted by $z_{i}$, and the number of nodes is shown in Figure 13. The proportional damping model is adopted:

$$
\mathbf{C}=\mu \mathbf{M}+\beta \mathbf{K},
$$

where $\mu$ and $\beta$ are proportional coefficients. In this case, $\mu$ is 4 and $\beta$ is $5 \times 10^{-5}$.

The FRFs of the original system are calculated for frequency band $0 \sim 200 \mathrm{~Hz}$ sampling at $0.1 \mathrm{~Hz}$, and natural frequencies $\omega_{r}$ are given in Table 6 . Assume that a lumped mass of $2 \mathrm{~kg}$ is placed in the node 6 . The modified natural frequencies $\omega^{*}{ }_{r}$ are obtained. As expected, the natural frequencies of the modified structure decrease. For example, the first mode's frequency shifts from $38.68 \mathrm{~Hz}$ to $36.06 \mathrm{~Hz}$. 
TABLE 7: Necessary spring coefficient $(\mathrm{N} / \mathrm{m})$ and the natural frequencies.

\begin{tabular}{lcccccc}
\hline Methods & Spring coefficient & 1 & \multicolumn{3}{c}{ Natural frequencies (Hz) } & \multicolumn{1}{c}{4} \\
\hline Original & & $\mathbf{3 8 . 6 8}$ & 78.87 & 117.16 & 175.71 & 195.51 \\
Optimal selection method & $k_{34(\mathrm{opt})}^{*}=5.82 \times 10^{4}$ & $\mathbf{3 8 . 5 9}(0.21 \%)$ & $73.38(3.15 \%)$ & $106.57(9.03 \%)$ & $160.07(8.91 \%)$ & $177.30(9.31 \%)$ \\
Absolute value method & $k_{34(\mathrm{abs})}^{*}=5.96 \times 10^{4}$ & $\mathbf{3 8 . 6 5}(0.06 \%)$ & $76.40(3.13 \%)$ & $106.57(9.03 \%)$ & $160.09(8.89 \%)$ & $177.31(9.30 \%)$ \\
\hline
\end{tabular}

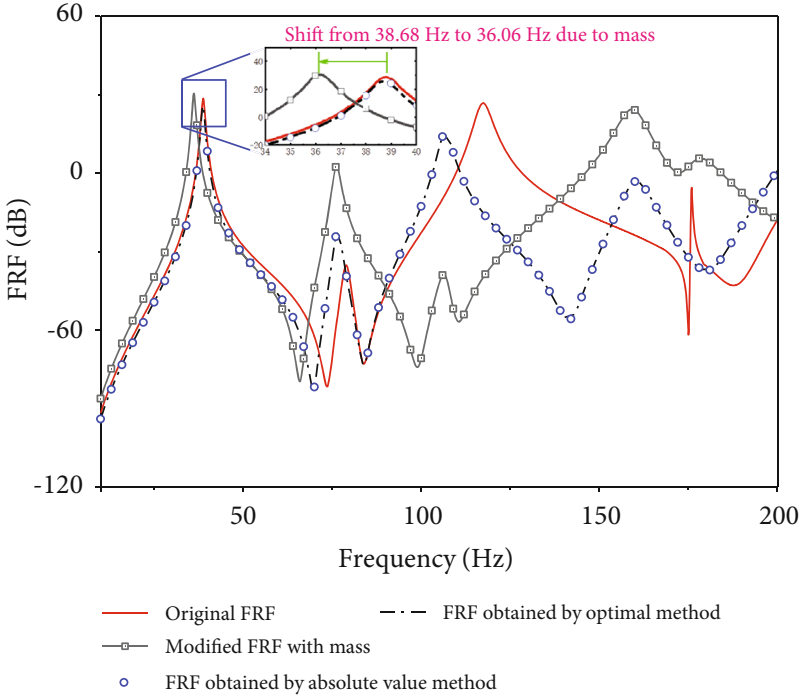

FIGURE 14: Comparison of original and modified FRF $h_{10,16}$.

A grounded spring is added in the $z_{16}$ direction to keep the first natural frequency unchanged. The value of the initial spring coefficient is $k^{*}=5.82 \times 10^{4}-1.29 \times 10^{4} i$. The stiffness determined by the proposed methods above is given in Table 7, respectively. Results show that the calculated stiffness values are slightly different between the optimal selection method and the absolute value method. The first natural frequency of the modified structure fits to the original value well, and the error between the modified value and the original value is negligible (only $0.06 \%$ ). The exact and modified FRF $h_{34}$ are shown in Figure 14. The FRF of the modified system near the first-order frequency range agrees well with the FRF of the original system.

\section{Conclusion}

To maintain a specific frequency of a damped system after mass modification, we propose herein the optimal selection method and the absolute value method. These two methods are implemented by installing a spring on the system. The efficiency and accuracy of the proposed methods are verified by the numerical simulations of the Six-DoFs damped system and a six-bay truss. The best spring location of the Six-DoFs damped system between the coordinates is determined. Results show that the optimal selection method has a similar capacity as the real method, which is only acceptable for the system with small damping. When the modal damping ratio reaches $4.24 \%$, the second-order error in the real method is up to $3.66 \%$, and that of the absolute method is only $0.83 \%$. Therefore, the absolute method has extensive applicability for not only low-level damping but also high-level damping.

\section{Data Availability}

The data used to support the findings of this study are available from the corresponding author upon request.

\section{Conflicts of Interest}

The authors declared no potential conflicts of interest with respect to the research, authorship, and/or publication of this article.

\section{Acknowledgments}

The authors are very grateful to the support of the National Natural Science Foundation of China (11602112 and 11572086), the Natural Science Foundation of Jiangsu Province (BK20170022), the Postgraduate Research \& Practice Innovation Program of Jiangsu Province (KYCX19_0062), and the Scientific Research Foundation of Graduate School of Southeast University.

\section{References}

[1] A. Kaveh, Optimal Structural Analysis, John Wiley \& Sons, 2014.

[2] D. Jiang, Y. Li, Q. Fei, and S. Wu, "Prediction of uncertain elastic parameters of a braided composite," Composite Structures, vol. 126, pp. 123-131, 2015.

[3] M. Nad, "Structural dynamic modification of vibrating systems," Applied and Computational Mechanics, vol. 1, no. 1, pp. 203-214, 2007.

[4] R. Zhu, Q. Fei, D. Jiang, and Z. Cao, "Removing mass loading effects of multi-transducers using Sherman-MorrisonWoodbury formula in modal test," Aerospace Science and Technology, vol. 93, article 105241, 2019.

[5] P. Zhang, Q. Fei, Y. Li, S. Wu, and Q. Chen, "Modal energy analysis for mechanical systems excited by spatially correlated loads," Mechanical Systems and Signal Processing, vol. 111, pp. 362-375, 2018.

[6] Y. Li, S. B. Mulani, R. K. Kapania, Q. Fei, and S. Wu, "Non-stationary random vibration analysis of structures under multiple correlated normal random excitations," Journal of Sound and Vibration, vol. 400, pp. 481-507, 2017.

[7] Y. Zhou, Q. Fei, and S. Wu, "Utilization of modal stress approach in random-vibration fatigue evaluation," Proceedings of the Institution of Mechanical Engineers, Part G: Journal of Aerospace Engineering, vol. 231, no. 14, pp. 2603-2615, 2017. 
[8] R. Belotti, D. Richiedei, and A. Trevisani, "Concurrent design of active control and structural modifications for eigenstructure assignment on a cantilever beam," in ASME 2017 International Design Engineering Technical Conferences and Computers and Information in Engineering Conference, Cleveland, OH, USA, August 2017.

[9] P. Olsson and P. Lidström, "Inverse structural modification using constraints," Journal of Sound and Vibration, vol. 303, no. 3-5, pp. 767-779, 2007.

[10] R. Zhu, Q. Fei, D. Jiang, and X. Hang, "Using ShermanMorrison theory to remove the coupled effects of multitransducers in vibration test," Proceedings of the Institution of Mechanical Engineers, Part G: Journal of Aerospace Engineering, vol. 223, no. 5, pp. 1364-1376, 2019.

[11] D. D. Sivan and Y. M. Ram, "Mass and stiffness modifications to achieve desired natural frequencies," International Journal for Numerical Methods in Biomedical Engineering, vol. 12, no. 9, pp. 531-542, 1996.

[12] T. Li and J. He, "Local structural modification using mass and stiffness changes," Engineering Structures, vol. 21, no. 11, pp. 1028-1037, 1999.

[13] M. Gürgöze and Ğ. S. Inceo, "Preserving the fundamental frequencies of beams despite mass attachments," Journal of Sound and Vibration, vol. 235, no. 2, pp. 345-359, 2000.

[14] Y. H. Park and Y. S. Park, "Structure optimization to enhance its natural frequencies based on measured frequency response functions," Journal of Sound and Vibration, vol. 229, no. 5, pp. 1235-1255, 2000.

[15] V. Mermertaş and M. Gürgöze, "Preservation of the fundamental natural frequencies of rectangular plates with mass and spring modifications," Journal of Sound and Vibration, vol. 276, no. 1-2, pp. 440-448, 2004.

[16] K. T. Joseph, "Inverse eigenvalue problem in structural design," AIAA Journal, vol. 30, no. 12, pp. 2890-2896, 1992.

[17] O. Çakar, "Mass and stiffness modifications without changing any specified natural frequency of a structure," Journal of Vibration and Control, vol. 17, no. 5, pp. 769-776, 2011.

[18] J. Sherman and W. J. Morrison, "Adjustment of an inverse matrix corresponding to a change in one element of a given matrix," The Annals of Mathematical Statistics, vol. 21, no. 1, pp. 124-127, 1950.

[19] Z. Liu, W. Li, H. Ouyang, and D. Wang, "Eigenstructure assignment in vibrating systems based on receptances," Archive of Applied Mechanics, vol. 85, no. 6, pp. 713-724, 2015.

[20] M. Huseyinoglu and O. Çakar, "Determination of stiffness modifications to keep certain natural frequencies of a system unchanged after mass modifications," Archive of Applied Mechanics, vol. 87, no. 10, pp. 1629-1640, 2017.

[21] F. J. Herrada, J. García-Martínez, A. Fraile, L. K. H. Hermanns, and F. J. Montáns, "A method for performing efficient parametric dynamic analyses in large finite element models undergoing structural modifications," Engineering Structures, vol. 131, pp. 625-638, 2017. 


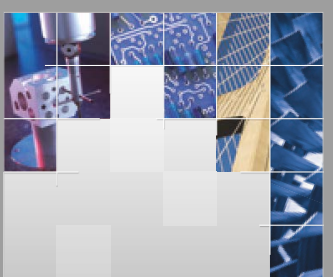

\section{Enfincering}
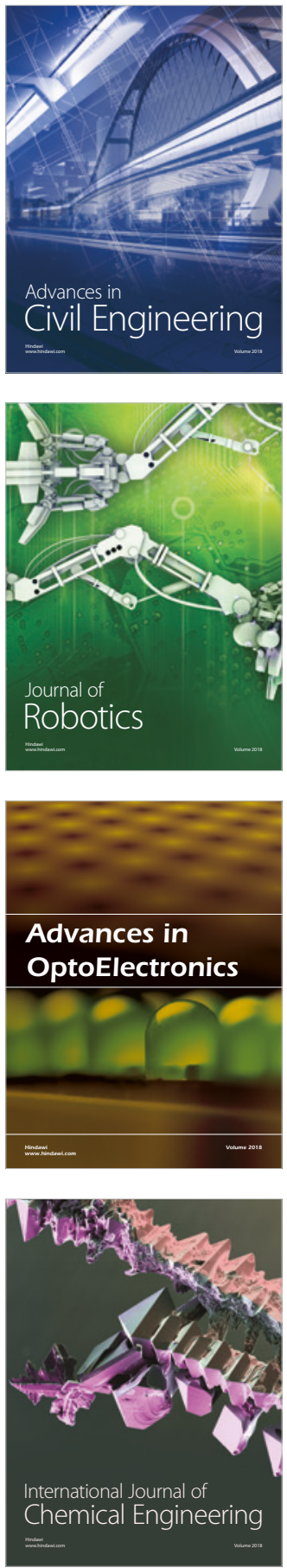

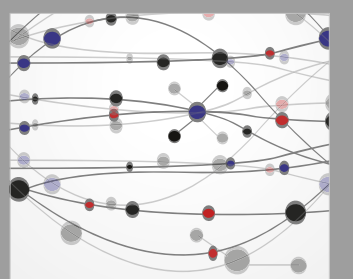

\section{Rotating \\ Machinery}

The Scientific World Journal

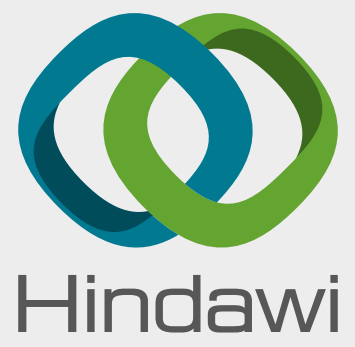

Submit your manuscripts at

www.hindawi.com
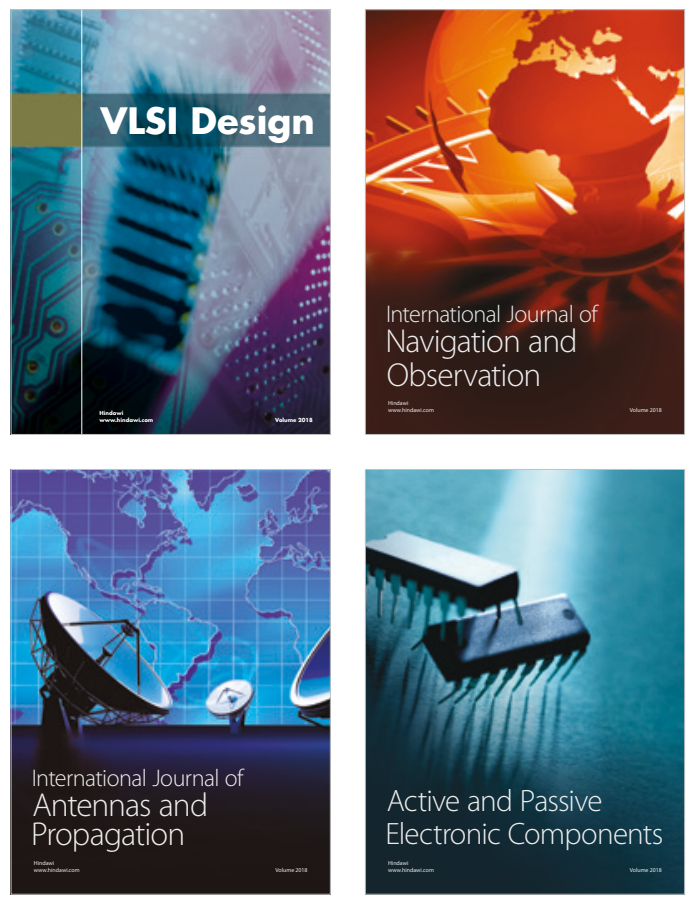
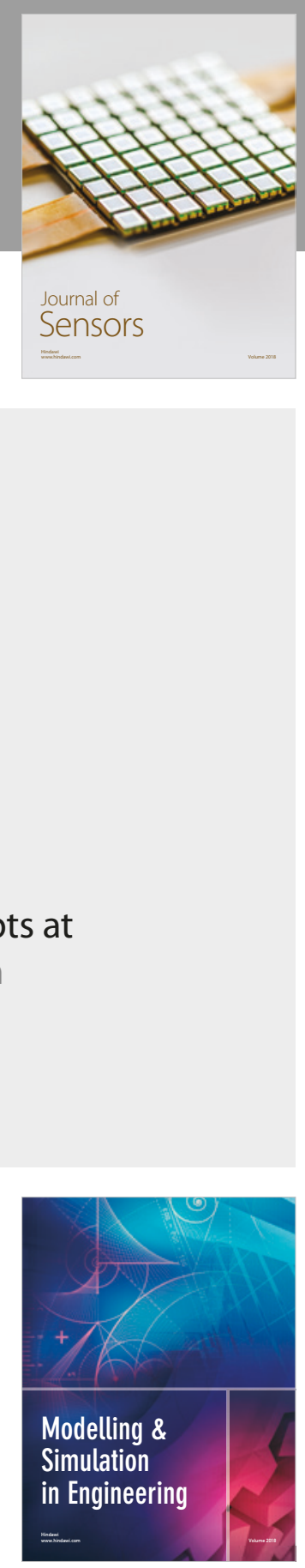

\section{Advances \\ Multimedia}
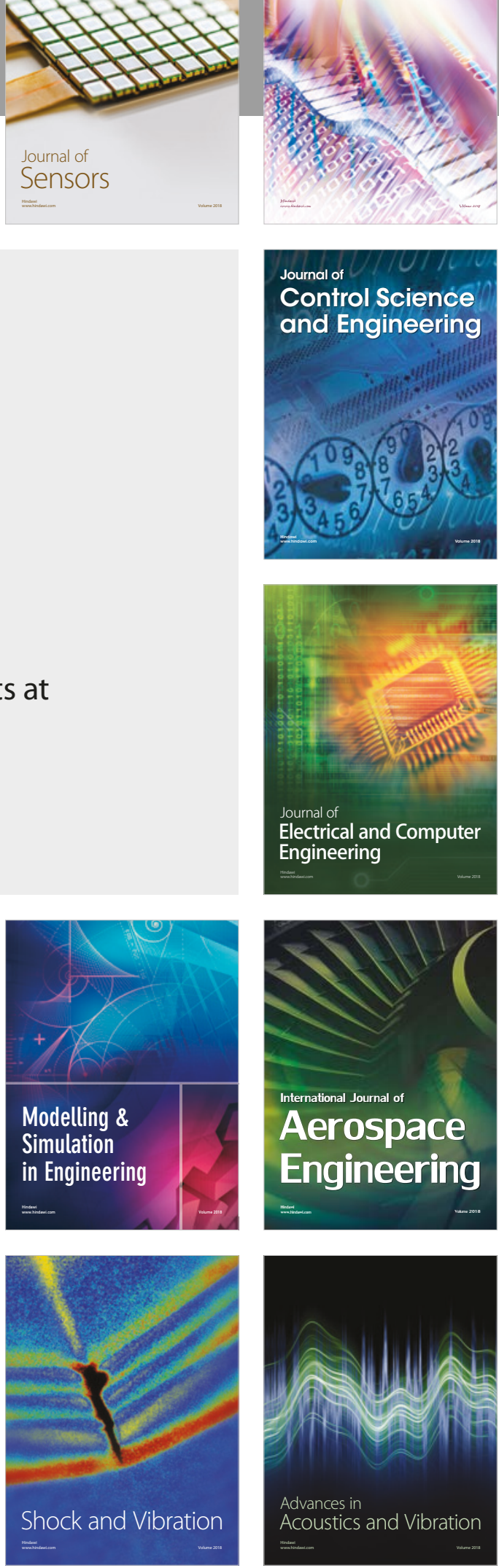